Voix et Images

volxetimages

\title{
De la mère patriarcale à la mère légendaire : Triptyque lesbien de Jovette Marchessault
}

\section{Lori Saint-Martin}

Volume 16, numéro 2 (47), hiver 1991

Jovette Marchessault

URI : https://id.erudit.org/iderudit/200897ar

DOI : https://doi.org/10.7202/200897ar

Aller au sommaire du numéro

Éditeur(s)

Université du Québec à Montréal

ISSN

0318-9201 (imprimé)

1705-933X (numérique)

Découvrir la revue

Citer cet article

Saint-Martin, L. (1991). De la mère patriarcale à la mère légendaire : Triptyque lesbien de Jovette Marchessault. Voix et Images, 16(2), 244-252.

https://doi.org/10.7202/200897ar d'utilisation que vous pouvez consulter en ligne.

https://apropos.erudit.org/fr/usagers/politique-dutilisation/ 


\title{
De la mère patriarcale à la mère légendaire: Triptyque lesbien de Jovette Marchessault
}

\author{
par Lori Saint-Martin, Ryerson Polytechnical \\ Institute
}

Continent noir $d u$ continent noir ${ }^{1}$, le rapport mère-fille est considéré par la plupart des théoriciennes féministes (Luce Irigaray, Nancy Chodorow, Carol Gilligan) comme le rapport au centre d'une vie de femme; peu étonnant alors que ce rapport se trouve au cœur de très nombreuses fictions de femmes. Si j'écris, c'est peut-être pour entendre à nouveau cette voix unique, continuer la chaîne parlée, parlante du langage des mères ${ }^{2}$, affirme Jovette Marchessault, dont toute l'œuvre porte l'empreinte de la mère. Dans ses romans, l'auteure met en scène une mère et une grand-mère "réelles", mais aussi des mères légendaires reliées à un passé matriarcal à redécouvrir, notamment la Grande-Oursonne, créatrice du ciel et de la terre ${ }^{3}$. Le théâtre de Jovette Marchessault fait revivre des écrivaines et des artistes du passé, autant de modèles à suivre, de mères spirituelles 4 .

Les trois courts textes qui composent Triptyque lesbien ${ }^{5}$ tournent également autour de la mère: mère rangée de la "Chronique lesbienne du moyen-âge québécois ", mère mi-soumise, mi-rebelle des "Vaches de nuit", mère libre des "Faiseuses d'anges". Tous ces textes doivent leur efficacité à la puissante convergence du réel et du

1 Luce Irigaray, le Corps-à-corps avec la mère, Montréal, les Éditions de la Pleine Lune, 1981, p. 61.

2 Jovette Marchessault, la Saga des poules mouillées, Montréal, les Éditions de la Pleine Lune, 1981, p. 21.

3 Gérald Gaudet, "La passion de l'impossible", dans. Voix d'écrivains, Montréal, Québec/Amérique, 1985, p. 126.

4 Ce sont nos mères, affirme simplement l'auteure (la Saga des poules mouillées, p. 24).

5 Jovette Marchessault, Triptyque lesbien, Montréal, les Éditions de la Pleine Lune, 1980. L'auteure écrit d'abord " tryptique ", mais rétablit l'orthographe standard dans les listes d'ouvrages parus. Le livre comprend les textes suivants: "Chronique lesbienne du moyen-âge québecois", "Les vaches de nuit ", "Les faiseuses d'anges *. 
mythique qu'illustre à merveille l'incipit du premier livre de l'auteure: Je suis d'origine céleste et je suis née dans les années trente 6.

La mère cosmique de Jovette Marchessault est nouvelle en littérature québécoise, sans précédent ou presque. On se rappellera toutefois que la grand-mère toute-puissante de la Route d'Altamont confectionne pour Christine éblouie une poupée à partir de rien, qui lui vaut d'être comparée à Dieu le Père. Ensuite, Christine est l'objet d'une vision:

[...] je dormais à demi [...] et voyais ma grand-mère arriver en colère au Paradis. Dans mon rêve, Dieu le Père, à la grande barbe et à l'air courroucé, céda la place à grand-mère aux yeux fins, rusés et clairvoyants. C'était elle qui, assise dans les nuages, dès lors prenait soin du monde, édictait de sages et justes lois. Or le pauvre monde sur terre s'en trouvait bien.

Longtemps il me resta dans l'idée que ce ne pouvait être un homme sûrement qui eût fait le monde. Mais, peut-être, une vieille femme aux mains extrêmement habiles. ${ }^{7}$

La grand-mère déesse de ce passage aux accents visionnaires, si inhabituels chez Gabrielle Roy, évoque la puissance créatrice des femmes et annonce les femmes légendaires ${ }^{8}$ de Jovette Marchessault. Peu étonnant alors que celle-ci célèbre Gabrielle Roy dans la Saga des poules mouillées.

\section{La théorie psychanalytique et la théorie féministe}

Triptyque lesbien s'inscrit dans un courant de réflexion féministe visant à penser le rapport mère-fille dans son contexte politique, symbolique, idéologique. Or toute analyse du rapport mère-fille doit passer obligatoirement par la théorie psychanalytique. Selon le modèle freudien, la petite fille vit d'abord avec sa mère une forme de fusion que Freud nommera tardivement la phase préœedipienne ${ }^{9}$. Lorsqu'elle se découvre démunie d'un pénis, le seul organe génital correct $^{10}$, elle en veut à sa mère de l'en avoir privée et se met à mépriser les femmes. Elle se tournera vers son père comme objet

6 Jovette Marchessault, Comme une enfant de la terre, Montréal, Leméac, 1975, p. 11.

7 Gabrielle Roy, la Route d'Altamont, Montréal, HMH, 1966, p. 30-31. Voir notre "Gabrielle Roy: The Mother's Voice, the Daughter's Text", a paraitre dans l'American Review of Canadian Studies.

8 Soyons légendaires! s'écrie Gertrude Stein dans Alice \& Gertrude, Natalie \& Renée et ce cher Ernest, Montréal, les Éditions de la Pleine Lune, 1984, p. 96.

9 Freud dit avoir sous-estimé dans un premier temps l'importance de cette période, qui peut durer quatre ou même cinq ans. Voir * Sur la sexualité féminine *, dans la Vie sexuelle, traduit de l'allemand par Denise Berger, Paris, PUF, 1969, p. 147.

10 Ibid., p. 149. 
d'amour et ensuite vers d'autres hommes. Bref, le lien entre mère et fille, fille et mère, doit être rompu pour que la fille devienne femme. La généalogie féminine doit être supprimée, au bénéfice de la relation filsPère, de l'idéalisation du père et du mari comme patriarches ${ }^{11}$.

La distance entre mère et fille est donc salutaire, nécessaire à la survie de la civilisation. Et si cette distance pouvait s'abolir? Si le rapport de la femme à la mère, le rapport des femmes entre elles ${ }^{12}$, était la grande inconnue de la psychanalyse? Au Québec, de très nombreux textes de femmes (qu'on pense à certains écrits de Louky Bersianik ou de Madeleine Gagnon) renvoient à cet espace préœdipien d'avant la rupture entre mère et fille. Dans Triptyque lesbien, le rapport mère-fille est fait de fusion, d'extase, d'une proximité source de joie et de force. Nous verrons que l'auteure cherche à remonter à une langue des origines matriarcales maintenant disparue: Je bondis sur le dos de ma mère. Je sens des mots oubliés se détacher de mes lèvres (p. 91).

\section{La mère patriarcale}

Si les petites filles s'éloignent de leur mère, diront les théoriciennes féministes, c'est par peur de devoir partager son statut dévalué et son peu de liberté ${ }^{13}$. Seules ou presque à s'occuper des enfants en bas âge, les mères enseignent à leurs filles la "féminité" traditionnelle qui leur permettra de s'intégrer à la société :

Ferme ta bouche quand tu manges. Viens dire bonjour à madame. Tais-toi quand les grandes personnes parlent. Mon dieu que t'as l'air négligée. N'oublie pas que tu es une future mère. 14

Bref, dans la pensée féministe radicale, la mère patriarcale, la parfaite dresseuse frustrée (p. 55), transmet à sa fille un héritage de honte et d'impuissance ${ }^{15}$ que celle-ci doit récuser afin de vivre pleine-

11 Luce Irigaray, Ethique de la différence sexuelle, Paris, les Éditions de Minuit, 1984, p. 106.

12 Luce Irigaray, Ce sexe qui n'en est pas un, Paris, les Editions de Minuit, 1977, p. 123.

13 Voir par exemple, Simone de Beauvoir, le Deuxième Sexe, tome 1, Paris, Gallimard, 1949, p. 305. Florentine, dans Bonheur d'occasion, est un excellent exemple de la jeune fille qui se promet une vie très différente de celle de sa mère et qui se désolidarise d'elle en pensée.

14 Dominique Gagnon, Louise Laprade, Nicole Lecavalier, Pol Pelletier, À ma mère, à ma mère, à ma mère, à ma voisine, Montréal, les Éditions du Remueménage, 1979, p. 18. Mary Daly, dans Gyn/Ecology: The Metaethics of Radical Feminism, Boston, Beacon Press, 1978, p. 139, 163-165, rappelle que ce sont les mères chinoises qui bandaient les pieds de leurs filles, les mères africaines qui pratiquent encore la clitoridectomie.

15 Gagnon et al., op. cit., p. 4. Voir aussi les rapports manqués entre Florentine et Rose-Anna. 
ment. Chez Jovette Marchessault, la mère patriarcale, agent spécial, déléguée par la centrale d'intelligence (p. 55) s'intègre au réseau scolaire, familial et social, dont la fonction est d'apprendre à la petite fille à se travestir en vraie femme (p. 16). En attendant d'être choisies par l'Homme, toutes les femmes, juments ou vaches du troupeau des ténèbres (p. 34), doivent se rassembler sur le trottoir ${ }^{16}$. Quand la narratrice voit surgir d'autres femmes qui marchent librement, en sens inverse, elle coupe les ponts avec celles $d u$ trottoir, y compris sa mère, et connaît un amour lesbien qui l'émerveille: Je venais de manger, je venais de boire en dehors de la mort ( $\mathrm{p}$. 77). Victime et bourreau involontaire, la mère patriarcale de la * Chronique *, dont la fille doit se détacher, s'efface ensuite au profit d'autres mères plus affranchies avec qui il fait bon vivre en symbiose.

\section{La mère marchandise}

Ma mère est une vache. Avec moi, ça fait deux (p. 83). Au fil du texte, l'injure courante se transforme et prend des connotations positives, grâce à une vibrante affirmation de solidarité mère-fille.

Selon Luce Irigaray relisant Marx et Lévi-Strauss, la société actuelle est fondée sur l'échange des femmes; celles-ci sont des "marchandises " qui font l'objet de transactions entre les hommes ${ }^{17}$. Or qu'arriverait-il, demande Irigaray, si les "marchandises» refusaient d'aller au "marché»? Entretenant entre elles un "autre» commerce? 18 "Les vaches de nuit" mettent en images cet *autre commerce", en jouant sur la confusion du sens propre et du sens figuré du mot "vache *. Mère et fille sont " réellement * (c'est-à-dire physiquement) des vaches; mais aussi "symboliquement" des vaches, des reproductrices.

Comme toutes les autres vaches, la mère est une bête de rente, un capital fixe (p. 84). Toutes ont été castrées en bas âge (p. 83), pour des raisons morales (elles sont naturellement nymphomanes) ou financières (castrées, elles sont plus soumises et plus productives). Bref, la vache de jour (p. 85) est une marchandise docile, privée de sexualité

16 Le trottoir des femmes, la rue des hommes sont des espaces symboliques qui évoquent les roles sociaux attribués en fonction du sexe. Voir Marthe Rosenfeld, "The Development of a Lesbian Sensibility in the Work of Jovette Marchessault and Nicole Brossard », dans Paula Gilbert Lewis, ed., Traditionalism, Nationalism and Feminism: Women Writers of Quebec, Westport, Greenwood Press, 1985 , p. 231.

17 Luce Irigaray, Ce sexe qui n'en est pas un, op. cit., p. 167.

18 Ibid., p. 193. Selon Andrea Dworkin, Right-Wing Women, New York, Perigee Books, 1978, p. 174, les hommes exploitent les femmes comme prostituées, selon le modele du bordel; comme reproductrices, selon le modele de la ferme. Jovette Marchessault montre les liens étroits entre les deux en plaçant le troupeau des femmes sur le trottoir. 
et de volonté propres. La nuit venue, la fille génisse est témoin d'une métamorphose: la mère change de robe (on dirait une gorgée de nuit étoilée, un soir de pleine lune [p. 35]), revêt sa fille d'une autre robe splendide et monte avec elle au ciel, pour assister au sabbat des sabots dans la voie lactée (p. 88). Toute la nuit, les mammiferes et les corneilles se remettent en mémoire une époque antérieure, le temps des mères (p. 93), temps de douceur, de fusion, d'amitié que le lien mère-fille recrée au présent. À l'aube, les vaches rentrent au bercail, sûres toutefois d'être bientôt libres: on se rapproche $d u$ moment où cette terre promise nous sera rendue (p. 94). Le texte est remarquable à la fois par ses images de vitalité et d'abondance ${ }^{19}$ et pour le retournement spectaculaire qu'il opère: d'insulte, vache devient un terme descriptif neutre, avant d'acquérir une connotation entièrement positive. Exemple frappant de revalorisation, par des féministes, d'images traditionnellement négatives de la femme ${ }^{20}$, permettant de repenser le passé et de proposer, des femmes, des visions nouvelles.

\section{La mère lesbienne}

Si le désir de la petite fille pour sa mère est un désir " phallique " (rappelons que, pour Freud, la libido est toujours masculine), il doit être supprimé au moyen d'un long conditionnement que Luce Irigaray nomme l'hétéro-sexualité normative ${ }^{21}$ et Nicole Brossard la convulsive habitude d'initier les filles au mâle comme une pratique courante de lobotomie ${ }^{22}$. Chez Jovette Marchessault, l'amour-fusion perdure entre la mère et sa fille adulte; dans ce contexte, le lesbianisme n'est pas un simple choix d'objet sexuel, mais bien un mode de vie fondé sur l'affirmation de soi et la complicité entre femmes, annonciateur d'un nouveau cycle de l'histoire ${ }^{23}$. Les seules femmes à vivre en dehors du troupeau des ténèbres sont les lesbiennes qui n'ont pas intériorisé le conditionnement social, survivantes obstinées du temps des mères. De même, mère et fille vivent ici une symbiose

19 Francine Noell, «Plaidoyer pour mon image ", Jeu, $n^{\circ} 16,3^{e}$ trimestre 1980 , p. 54, écrit que les Vaches de nuit marquent un point de non-retour dans l'onirisme féminin québécois [...] le texte ouvre toute grande la scène à une cohorte puissante et ravageante de fortes femmes, dorénavant irrémédiablement présentes et dont le deferlement est impossible à contenir.

20 Autres exemples dans l'œuvre de) Jovette Marchessault, les poules mouillées (voir Marguerite Le Clézio, «Poétique et/ou politique: le théâtre de Jovette Marchessault „, North Dakota Quarterly, vol. LII, $n^{\circ} 3$, été 1984, p. 103) et les faiseuses d'anges. Sur la revalorisation de la sorcière, voir notre «Ecriture et combat féministe: figures de la sorcière dans l'écriture des femmes au Québec », à paraitre dans Québec Studies.

21 Luce Irigaray, le Corps-à-corps avec la mère, op. cit., p. 31.

22 Nicole Brossard, l'Amèr, Montréal, l'Hexagone, 1988, p. 109 (Typo).

23 Gloria Feman Orenstein, "Jovette Marchessault, the Ecstatic Vision-Quest of the New Feminist Shaman *, dans Barbara Godard, ed., Gynocritics/la Gynocritique, Toronto, ECW Press, 1987, p. 189-190. 
qui fait d'elles des amantes: Un double enchantement nous lie l'une à l'autre, dans un seul corps [...] (p. 86). Tandis que les penseurs patriarcaux joignent à une vénération pour la pureté de la mère, un dégoût profond pour son corps ${ }^{24}$ (inter foeces et urinam nascimur, écrit saint Augustin), le corps de la mère est célébré ici par une fille lesbienne qui en connaît tous les replis:

Je visite ma mère mamelle, ma mère fruit, ma mère plante, ma mère écailles et viscères [...] Je vois son âme, sa vulve, sa matrice, sa rosée sauvage, son nectar rose, l'éclair minéral de ses grottes. Je touche à tout ! Je goûte à tout! (p. 89)

Jovette Marchessault recherche, selon la formule de Luce Irigaray, les paroles qui disent le rapport à la fois le plus archaïque et le plus actuel au corps de la mère ${ }^{25}$. Ici, le sang de la vie (le lait virant au rouge et son scandaleux secret de sang [p. 84]; la mère qui tricote du rouge [p. 102]) n'inspire plus le dégoût mais le ravissement. Autre image d'abondance, le lait maternel:

Le lait coule! Le lait gicle! Le lait coule à flot! Beauté, beauté, bonté blanche. Le lait neige! La lait goutte, le lait odore! Le lait poudre! Le lait rafale! Le lait ouragane! [...] L'implacable soif des filles invite le lait à se répandre dans les hautsfonds du ciel... (p. 87) ${ }^{26}$

Selon Freud, presque tous les enfants reprochent à leur mère de les avoir sevrés trop tôt, les laissant pour toujours non rassasiés ${ }^{27}$. La mère lesbienne cosmique, elle, peut aller jusqu'au bout de la soif, offrir à la fille tout l'amour qu'elle recherche. Car dans ce rapport idéalisé, aucune ambivalence: il est don, paix, abolition de la distance préconisée par la théorie psychanalytique. La réciprocité mère-fille élimine en douceur la mère patriarcale qui contrôle et surveille et instaure des relations égalitaires: fille, mère, mère, fille, la hiérarchie s'en va faire un tour ailleurs quand les vaches de nuit se bercent (p. 87) ${ }^{28}$.

Dans de nombreux écrits féministes, surgit ce qu'Adrienne Rich appelle la matrophobie ${ }^{29}$, un désir de fuir la féminité traditionnelle

24 Adrienne Rich, Of Woman Born: Motherhood as Experience and Institution, New York, Norton, 1976, p. 34.

25 Luce Irigaray, le Corps-à-corps avec la mère, op. cit., p. 28-29.

26 Ce passage serait à rapprocher de celui où Hélène Cixous évoque une écriture féminine à l'encre blanche: Un enfant! Du papier! Iuresses! Je déborde! Mes seins débordent! Du lait. De l'encre [...] Le goat de lait de l'encre (la Venue à l'écriture, Paris, Union générale d'éditions, 1977, p. 37 (10/18).

27 Sigmund Freud, loc. cit., p. 147.

28 II faudrait pouvoir établir avec nos mères un rapport de réciprocité de femme à femme, ou elles pourraient aussi éventuellement se-sentir nos filles, écrit Luce Irigaray (le Corps-à-corps avec la mère, op. cit., p. 86).

29 Adrienne Rich, op. cit., p. 236. 
qu'incarne la mère et de mener une vie libre ${ }^{30}$. Mais chez Jovette Marchessault, la mère n'est pas liée à l'impuissance et promise à la mort ${ }^{31}$; au contraire, mère et fille sont liguées contre l'impuissance et décidées à vivre heureuses ensemble. Loin de blâmer sa mère d'être párfois une vache de jour, la fille la comprend et affirme une fois de plus l'amour qui les lie:

Détester sa mère - comme certaines ont pu le précòniser - ne sort pas de la soumission : la haine n'est que l'autre versant de la soumission. Nos ressentiments face à nos mères ne peuvent nous réussir puisque la culture nous a justement appris à rompre avec elles pour aimer les hommes, en y perdant notre identité sexuelle [...] Il est donc plutôt question de trouver son identité de femmes, dans et avec sa mère. ${ }^{32}$

\section{La mère légendaire}

À partir du couple mère-fille, s'élève un modèle de solidarité entre femmes qui est source de puissance et de combativité: L'héritage génétique de mes mères faisait que j'avais encore dans la souvenance, le ventre, le cœur, une maudite épaisseur de résistance (p. 20). Par le biais de la mère, on renoue avec la grande déesse primordiale, la toute belle, la mère du premier vice, l'immense grand-mère de la Terre et du ciel (p. 57); inversement, du fait qu'elle participe de la tradition matriarcale ${ }^{33}$, la mère devient à son tour une immense figure légendaire :

Elle est plus grande que les géants, ma mère! (p. 89)

Ma mère est une légende [...] Ma mère existe. [...] Ma mère existe et cela me met en extase. (p. 104)

La mère faiseuse d'anges est une révoltée dont le point de tricot se transforme en poing (p. 102). Son tricot n'est pas un inoffensif ouvrage de dame, mais bien un acte d'affirmation de soi dont la portée est cosmique: Je sens circuler dàns les laines de ma mère un orage universel (p. 102). En délivrant une femme d'une grossesse non désirée, elle l'aide à trouver une nouvelle identité bien à elle: elles se tricotent un avenir [...] elles s'aident à monter à pas de louve jusqu'au

30 Je regarde ma mère et jai envie de vomir [...] la honte de faire partie d'un troupeau d'esclaves, affirme Pol Pelletier dans l'ouvrage collectif, la Nef des sorcières, Montréal, Quinze, 1976, p. 67.

31 Dans Ầ ma mère, à ma mère, à ma mère, à ma voisine, on assiste à la mise à mort de la mère patriarcale; dans l'Amèr, op. cit., Nicole Brossard écrit: Jai tué le ventre et je l'écris (p. 26).

32 Carole Henry et Francine Pelletier, "Luce Irigaray: un message amoureux", la Vie en rose, juin-juillet-aout 1982, p. 64 .

33 Certains historiens contestent l'existence d'une epoque matriarcale; mais ce qui compte, c'est qu'elle est une source d'inspiration pour de très nombreuses femmes. 
cœúr pour que surgisse encore l'espérance (p. 110). Sa vie offre à sa fille des images de beauté et de résistance qui la vivifient à son tour.

Toutes les mères légendaires de Jovette Marchessault, de la Mère des herbes à Anaïs Nin en passant par la vache de nuit, rappellent l'histoire enfouie, oubliée des femmes. Sortir du bois de l'amnésie patriarcale ${ }^{34}$, à la fois envahir la légende et le mythe et l'Histoire (la Saga des poules mouillées, p. 171), tel est le projet de Jovette Marchessault, qu'elle réalise par le biais de ses mères à la fois mammifêres et humaines, mythiques et réelles, célestes et terrestres ${ }^{35}$.

Marquée elle aussi par une fusion entre femmes, l'époque matriarcale n'est pas sans lien avec la période préœdipienne qui devient, chez de nombreuses écrivaines, un espace mythique dont le prolongement s'observe dans les rapports idéalisés entre femmes, notamment entre mères et filles ${ }^{36}$. C'est dans la phase préœdipienne qu'existerait un parler-femme, une symbiose parfaite entre mère et fille, dont la nostalgie habite la femme adulte ${ }^{37}$. Par la revalorisation d'injures et d'images stéréotypées (la vache, la faiseuse d'anges), par la féminisation et le télescopage d'expressions toutes faites (entre chienne et louve [p. 93]; mes doigts de fée du logis [p. 54]), par les énumérations exubérantes et l'invention verbale (la trinité bullière [p. 20]; la transmission automatique, de père en fils [p. 59]), Jovette Marchessault travaille à l'élaboration d'un langage féminin qui prend origine dans la mère.

Les femmes légendaires de Jovette Marchessault ont inspiré à la critique certaines réserves. Monique Dumont affirme que la mythologie païenne qu'elle évoque a pour résultat, tout comme la chrétienne, une déification de la féminité. Mais les femmes gagnent-elles encore à être déifiées? ${ }^{38}$ L'usage du mythe est-il en soi libérateur ? ${ }^{39}$, demande Thérèse Marois. Oui, le mythe est potentiellement réducteur; mais une si longue tradition rabaisse le féminin, qu'il n'est pas inutile de créer quelques mythes de femmes fortes, vivantes, plus grandes que nature. Certes, la mythologie et la littérature ne manquent pas de femmes fortes - Médée, Circé, lady Macbeth -, mais elles sont généralement destructrices, meurtrières, tandis que chez Jovette Marchessault, la puissance est source de vie et d'illumination.

34 Jovette Marchessault, «Nous n'écrivons plus pour les fonds de tiroirs», Châtelaine, vol. $X X, n^{\circ} 1$, janvier 1979, p. 69.

35 Gloria Femán Orenstein, "Postface ", dans Triptyque lesbien, op. cit., p. 119.

36 Sur la phase préedipienne, voir Marianne Hirsch, The Mother/Daughter Plot: Narrative, Psychoanalysis, Feminism, Bloomington, Indiana University Press, 1989, p. 133.

37 C'est aussi sur cette phase que se penchent en général les théoriciennes du développement moral des femmes.

38 Monique Dumont, *Une filiation d'ecriture *, Spirale, $n^{\circ} 10$, juin 1981, p. 19.

39 Therèse Marois, «Mythes féministes: la Saga des poules mouillées au T.N.M.», Jeu, vol. $X, n^{\circ} 20,1981$, p. 54. 
À condition de ne pas s'appuyer sur une prétendue supériorité ontologique des femmes, le mythe de la déesse-mère propose aux femmes une image de force et de vitalité qui peut être source de nouveaux dynamismes. Ce mythe revêt d'ailleurs une importante dimension politique, en ce sens qu'il valorise la force et l'autonomie des femmes. Le mythe descend dans la rue, si on peut dire, en même temps qu'il monte jusqu'à la Voie lactée. La déesse, chez Jovette Marchessault, est aussi une femme liée d'amour à sa fille et aux autres femmes, une femme sexuée dont on célèbre le corps, pas un pur esprit, un modèle inaccessible comme la Sainte Vierge. Toute femme énergique et vivante peut en observer la trace en elle. Qu'on soit lesbienne ou non, les relations utopiques entre mères et filles que dépeint Jovette Marchessault réjouissent et font rêver.

Liée au mensonge 40 ou au conditionnement social dans d'autres textes lesbiens, la mère, chez Jovette Marchessault, est mémoire, retour aux origines, solidarité. Située à mi-chemin entre un paradis perdu et un futur utopique à faire advenir, elle est celle par qui on échappera au présent oppressif. Prépatriarcales, préœedipiennes, femmes de chair et de mots, les mères chez Jovette Marchessault permettent de renouer avec une généalogie féminine ${ }^{41}$ oubliée et d'imaginer de nouveaux rapports entre femmes, à l'orée d'un autre monde.

40 Th me mens me mens maman, se lamente la petite fille dans $A$ ma mère, à ma mère, à ma mère, à ma voisine, op. cit., p. 15.

41 Luce Irigaray, le Corps-à-corps avec la mère, op. cit., p. 30. 